\title{
Ultrasonography for lumbar neuraxial block
}

\author{
Seokha Yoo, Youngwon Kim, Sun-Kyung Park, Sang-Hwan Ji, and \\ Jin-Tae Kim
}

Recieved August 3, 2020

Accepted August 20, 2020

\author{
Corresponding author \\ Jin-Tae Kim, M.D., Ph.D. \\ Department of Anesthesiology and \\ Pain Medicine, Seoul National \\ University Hospital, Seoul National \\ University College of Medicine, 101 \\ Daehak-ro, Jongno-gu, Seoul 03080, \\ Korea \\ Tel: 82-2-2072-3295 \\ Fax: 82-2-747-8363 \\ E-mail: jintae73@gmail.com
}

Department of Anesthesiology and Pain Medicine, Seoul National University Hospital, Seoul National University College of Medicine, Seoul, Korea

\begin{abstract}
Ultrasonography can be useful to perform a lumbar neuraxial block. It aids in understanding the anatomy of the lumbar spine before the procedure. Preprocedural ultrasound imaging provides information about the accurate intervertebral level for puncture, optimal needle insertion point, and depth of needle advancement for a successful neuraxial block. The key ultrasonographic views for lumbar neuraxial block include the transverse midline interlaminar and parasagittal oblique views. Ultrasonography can facilitate lumbar neuraxial block in difficult cases, such as the elderly, obese patients, and patients with anatomical abnormality of the lumbar spine. This review elucidates the basics of spinal ultrasonography for lumbar neuraxial block and the current evidence regarding ultrasound-guided neuraxial block in adults.
\end{abstract}

Keywords: Anesthesia, epidural; Anesthesia, spinal; Lumbar vertebrae; Ultrasonography.

\section{INTRODUCTION}

Recently, the use of ultrasonography has become popular in operating rooms. The lumbar neuraxial block was traditionally performed using a surface landmark-guided technique. However, ultrasound (US)-guided technique has been more frequently used for neuraxial block. This article reviews the sonoanatomy of the lumber spine, US-guided techniques for neuraxial block, and current evidence for the clinical usefulness of US-guided lumbar neuraxial block.

\section{GROSS ANATOMY OF THE LUMBAR VERTEBRAE}

The lumbar vertebra is composed of the vertebral body, pedicle, transverse process, superior articular process, inferior articular process, lamina, and spinous process. The gaps between two adjacent vertebrae can be divided into the interspinous and interlaminar spaces. The interlaminar space is bounded by the bases of the spinous processes, laminae, inferior articular processes, and superior articular processes (Fig. 1). For successful dural puncture, the spinal needle should be entered through the interlaminar space.

\section{SONOANATOMY OF THE LUMBAR VERTEBRAE}

To obtain ultrasonographic view of the lumber spine, a curved-array probe is placed on the patient's back in sitting or lateral decubitus position with lumbar spine flexion. An US probe can be applied in three basic ways: sagittal, transverse, and diagonal views. The angle of the probe can be adjusted medially in the parasagittal plane or tilted cephalad or caudad in the transverse plane to obtain the best image of the target structures. Although the diagonal view is not commonly used for preprocedural US imaging, it can be used for real-time US-guided neuraxial block.

This is an Open Access article distributed under the terms of the Creative Commons Attribution Non-Commercial License (http://creativecommons.org/licenses/by-nc/4.0) which permits unrestricted non-commercial use, distribution, and reproduction in any medium, provided the original work is properly cited.

Copyright (C) the Korean Society of Anesthesiologists, 2020 


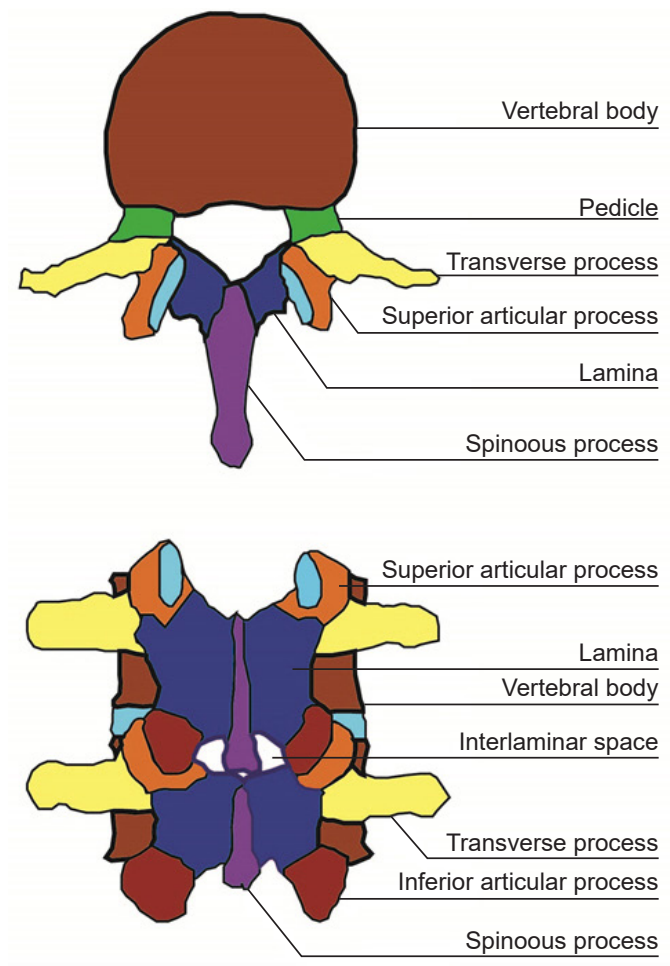

Fig. 1. Lumbar vertebrae anatomy.

\section{SAGITTAL VIEWS OF THE LUMBAR SPINE}

There are five basic sagittal plane views of the lumbar spine according to the probe location and direction. By moving the probe from a lateral position to the midline of the neuraxis, sagittal transverse process, sagittal articular process, sagittal lamina, and sagittal spinous process views can be obtained (Fig. 2A-D). From the probe position having the sagittal articular process view or sagittal lamina view, the parasagittal oblique view can be obtained by tilting the probe medially towards the midline (Fig. 2E). The parasagittal oblique view can be used for the determination of optimal intervertebral level for puncture by identifying the intervertebral level at which the posterior complex (ligamentum flavum-dura complex) and the anterior complex (the posterior longitudinal ligament, posterior surface of the vertebral body, and intervertebral disc) are visualized most clearly. It is also useful to select the intervertebral level at which the interlaminar height is the largest.

\section{TRANSVERSE VIEWS OF THE LUMBAR SPINE}

There are two basic transverse views for lumbar neuraxial block: transverse spinous process view and transverse

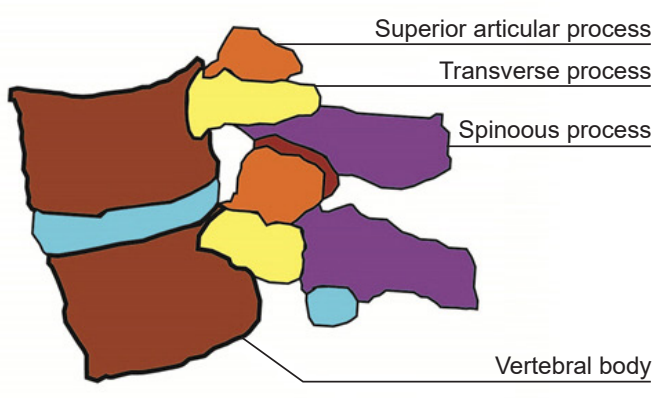

interlaminar view. The transverse spinous process view is used to determine the midline composed of connecting spinous process tips (Fig. 3A). The transverse interlaminar view can be obtained by sliding the probe in a cephalad or caudad direction from the transverse spinous process view (Fig. 3B). Slight cephalad or caudad tilt in the transverse interlaminar view may be needed to obtain the image showing the dural sac located between the anterior and posterior complexes (Fig. 3C).

\section{DIAGONAL VIEW OF THE LUMBAR SPINE}

The diagonal view can be obtained by rotating the probe approximately 45 degrees from the sagittal articular process view, resulting in a combination of transverse and sagittal views. In this view, the spinous process of the upper vertebral body, interlaminar space, and lamina of the lower vertebral body can be visualized. It can be used for real-time US-guided neuraxial block (Fig. 4).

\section{US-GUIDED TECHNIQUES FOR LUMBAR NEURAXIAL BLOCK}

A systematic approach to US-guided lumbar neuraxial block in adults has been well-described in a previous re- 

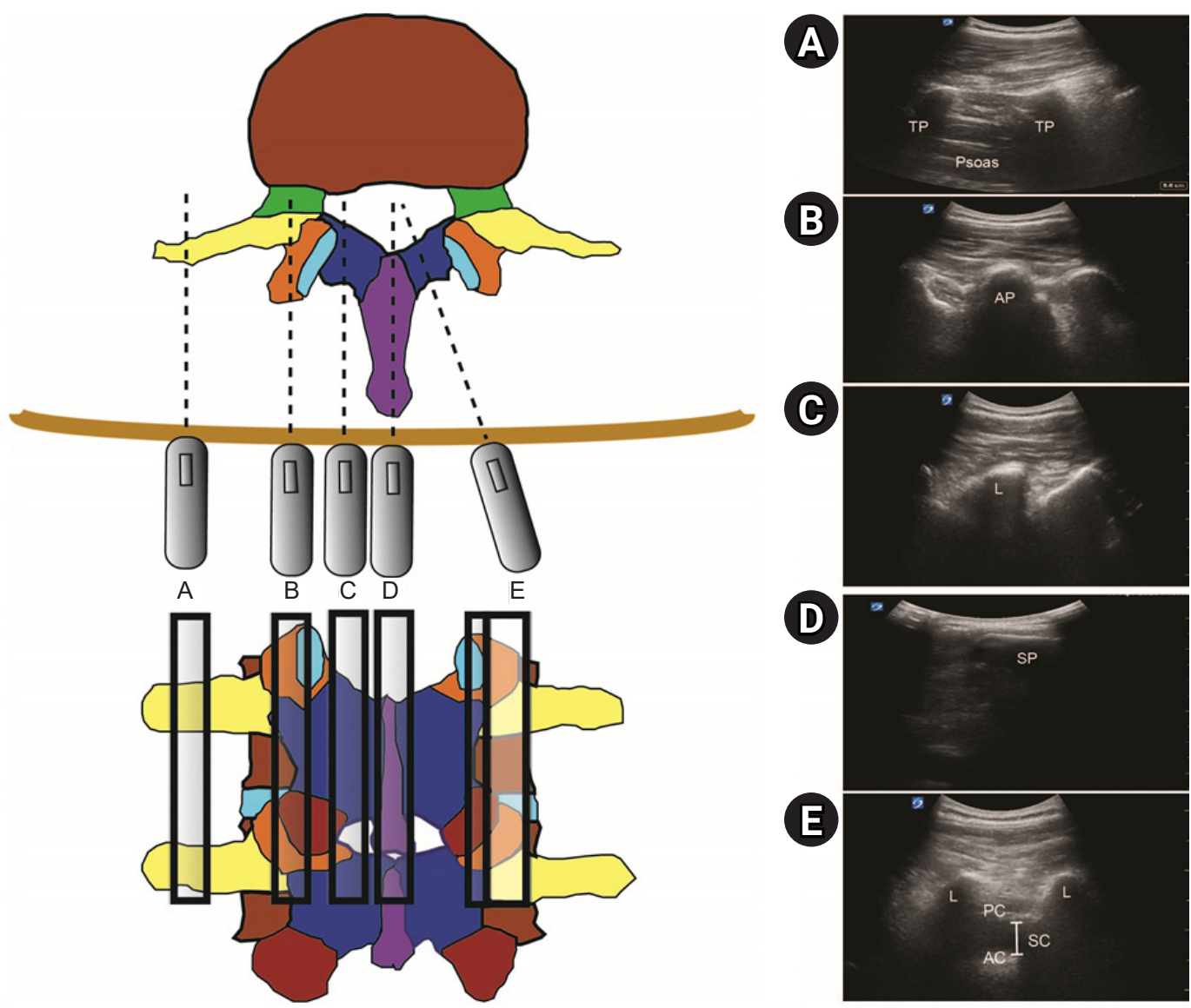

Fig. 2. Sagittal views of the lumbar spine. (A) Sagittal transverse process view, (B) sagittal articular process view, (C) sagittal lamina view, (D) sagittal spinous process view, (E) parasagittal oblique view. TP: transverse process, AP: articular process, L: lamina, SP: spinous process, PC: posterior complex, AC: anterior complex, SC: spinal canal (intrathecal space).
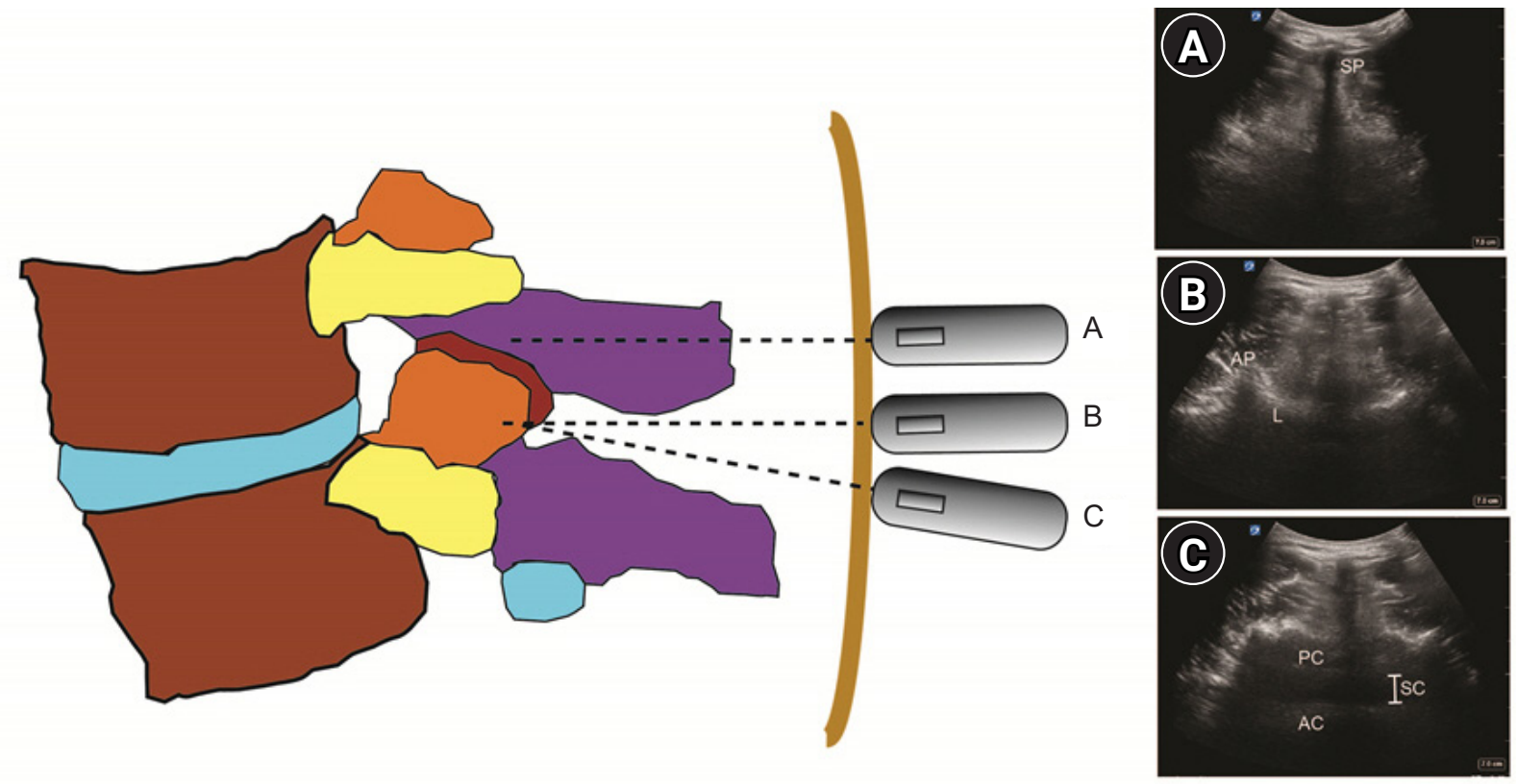

Fig. 3. Transverse views of the lumbar spine. (A) Transverse spinous process view, (B) transverse interspinous process view, (C) tilted transverse interspinous process view. SP: spinous process, AP: articular process, L: lamina, PC: posterior complex, AC: anterior complex, SC: spinal canal (intrathecal space). 


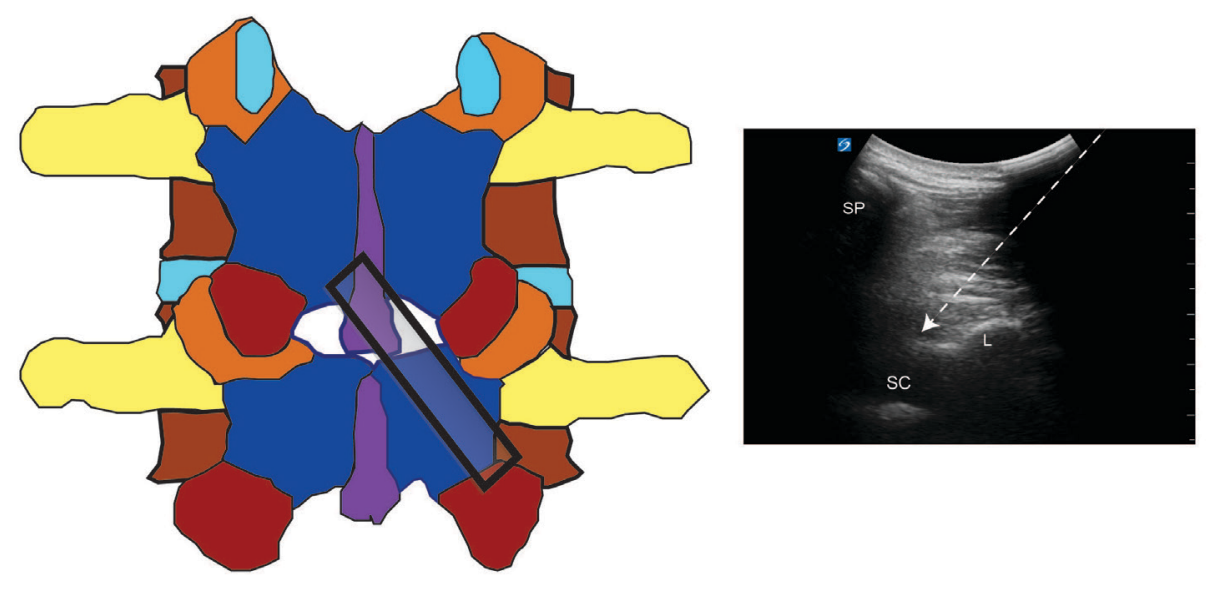

Fig. 4. Diagonal view of the lumbar spine. Probe is applied over the spinous process of the upper lumbar vertebra and lamina of the lower lumbar vertebra. SP: Spinous process, SC: spinal canal, L: lamina. Arrow: pathway of spinal needle.

view article [1].

Ultrasonography can be used in two basic ways for lumbar neuraxial block: preprocedural US scanning or real-time US-guidance. A low-frequency (e.g., 2-5 MHz), curved-array US probe is usually used. To optimize sonographic images, adjustment of depth (usually $7-10 \mathrm{~cm}$ ), focus positioning, and gain settings on the US machine are essential. During US-guided neuraxial block, it is crucial to remove gel or chlorhexidine from the skin before needle insertion to avoid potential neurologic complications, including adhesive arachnoiditis $[2,3]$.

\section{Preprocedural US-assisted midline approach}

1. Confirm the midline based on the spinous processes by placing the US probe over the midline in a horizontal orientation (the transverse spinous process view).

2. Locate the interlaminar space using the parasagittal oblique view or transverse interlaminar view and choose the most appropriate intervertebral level for neuraxial puncture.

3. Determine the needle insertion point and angle of needle trajectory using the transverse interlaminar view. The US probe can be tilted cephalad or caudad to visualize the intrathecal space. Remember the three-dimensional angle of the probe where the posterior and anterior complexes are visualized most clearly.

4. Estimate the depth of needle insertion by measuring the distance from the skin to the posterior complex.

5. Perform neuraxial block by inserting a needle at the predetermined insertion point with the insertion angle.

\section{Preprocedural US-assisted paramedian approach based on bony structures}

1. Confirm the neuraxial midline based on the spinous processes as per the transverse spinous process view.

2. Locate the interspinous space using the transverse view. If possible, identify the interlaminar space using the parasagittal oblique view and select the intervertebral level for neuraxial puncture.

3. Having identified the midline, spinous process, and interlaminar space, insert a spinal needle at the point approximately $1 \mathrm{~cm}$ superior to the lower spinous process and $1 \mathrm{~cm}$ lateral to the midline, or at the point approximately $1 \mathrm{~cm}$ inferior to the caudad tip of the upper spinous process and $1 \mathrm{~cm}$ lateral to the midline.

4. Slight medial (5-10 degree) and cephalad (5-10 degree) angulation of needle insertion may be needed similar to a conventional paramedian approach.

The abovementioned approach is similar to the conventional paramedian approach in dependence on the location of key bony structures. However, with the help of a US scan, more precise identification of underlying bony structures is possible. This approach can be useful in extremely obese patients or when the quality of US images is inadequate.

\section{Preprocedural US-assisted paramedian approach based on the parasagittal oblique view}

1. Confirm the midline in the transverse spinous process view and apply the probe in a longitudinal direction $1-2 \mathrm{~cm}$ lateral to the midline with a slight medial tilt. 
2. Identify the interlaminar space in the parasagittal oblique view and select the intervertebral level that provides the largest interlaminar space.

3. Determine the medial angle of the sagittal plane providing the clearest image of the interlaminar space. Slight cephalad or caudad angulation of the probe may be necessary in some cases.

4. Estimate the depth of needle insertion by measuring the distance from the skin to the posterior complex.

5. Insert a needle at the designated insertion point with the designated angle.

Paramedian approach based on the parasagittal oblique view has potential advantages over the midline approach using the transverse interlaminar view because the parasagittal oblique view provides better visibility of the interlaminar space than the transverse interlaminar view, especially in the elderly. When the US beam reaches the spinal canal in the parasagittal oblique view, the needle can also reach the canal through the same pathway. When using US-assisted paramedian approach, cephalad or caudad needle angulation may not be required. This approach can be the most direct way to the intrathecal or epidural space through the interlaminar space considering only medial angulation.

\section{Real-time US-guided neuraxial block}

Real-time US-guided neuraxial block is a feasible and promising technique that can result in successful neuraxial anesthesia in difficult cases $[4,5]$. However, it is tricky to perform because of the large size of the probe, small gauge of the needle, and relatively deep target structure. There are several methods to perform real-time US-guided neuraxial block, including sagittal, transverse, and diagonal in-plane approaches.

Real-time US-guided spinal anesthesia using in-plane approach based on the parasagittal oblique view can increase first-attempt success rate compared to the landmark-guided paramedian approach technique [6]. Needle approach from the non-dependent side may lead to dry tap due to gravity, even if the needle tip is placed in the intrathecal space. A prospective observational study showed that real-time US-guided spinal anesthesia using in-plane approach based on the diagonal view was successfully performed in 97 out of 100 consecutive patients within three median needle passes [7]. Probe application site can be slightly moved to secure the room for puncture site and needle manipulation during the transverse in-plane paramedian approach [8]. Electromagnetic needle tracking system can also be used for real-time US-guided spinal anesthesia [9].

\section{US-guided neuraxial block in patients with scoliosis}

Preprocedural US assistance may have potential benefits in neuraxial block for patients with scoliosis. Systematic algorithms to guide neuraxial techniques in patients with scoliosis have been described previously $[10,11]$. Several earlier publications have demonstrated that the use of ultrasonography is useful for spinal anesthesia in patients with scoliosis $[5,12,13]$. The lateral curvature of the scoliotic spine can be confirmed by marking out all spinous process tips using ultrasonography. Simple spinal radiographs or computed tomography are also helpful. In addition to the lateral curvature, rotational change of the vertebral body should be considered when performing neuraxial block in these patients. During the paramedian approach, it is easier to insert a needle on the convex side of the vertebral column after confirming the spinous process considering the needle insertion angle. For example, in the paramedian approach, if the rotation of the vertebral body is approximately 15 degrees in a patient with scoliosis, the needle insertion site is on the convex side of the spinous processes, therefore, the angle of needle trajectory would be perpendicular to the skin towards the interlaminar space (Fig. 5A). On the other hand, when using the midline approach through the interspinous space in a patient with scoliosis, the angle of needle insertion would be 15 degrees off the sagittal plane towards the convex side (Fig. 5B).

\section{USEFULNESS OF US-GUIDED NEURAXIAL BLOCK}

US imaging can provide important clinical information for a successful neuraxial block. Ultrasonography aids in identification of the accurate puncture level by providing information, such as the widest inter-laminar space, depth to the dura from the skin, and accurate spinal level.

To achieve successful neuraxial blockade, accurate identification of the intervertebral spaces is crucial. US imaging is also useful in localizing the intervertebral spaces and identifying lumbar vertebral level. Although many anesthesiologists used to identify the vertebral level by palpation when performing neuraxial blockade, previous studies 


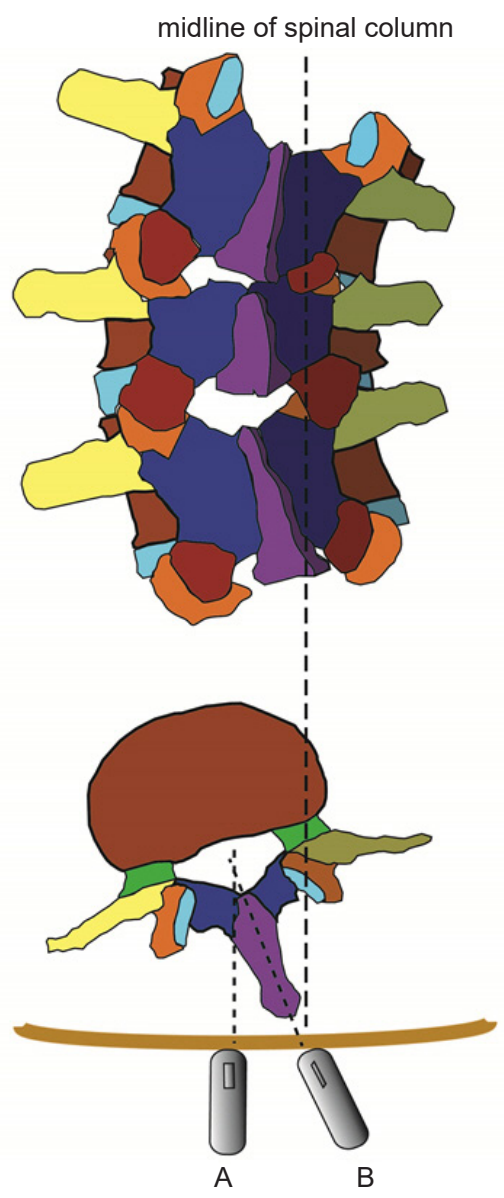

Fig. 5. Ultrasound scans for patients with scoliosis. (A) Paramedian approach. (B) Midline approach.

consistently showed that identification of the intervertebral level using palpation is unreliable [14-17]. Preprocedural neuraxial US imaging not only provides the anatomical details of the intervertebral space and bony structures but also the optimal skin puncture point and needle insertion angle, and these are valuable for improving the ease of performing neuraxial blockade $[18,19]$.

Preprocedural neuraxial US imaging can facilitate dural puncture or epidural catheterization by predicting the distance from the skin to the epidural or intrathecal space. Many studies demonstrated that US-determined depth to the epidural or intrathecal space was well-correlated with the actual needle depth [20-23]. Moreover, the information on the distance from the skin to the epidural space can decrease the rate of failed labor epidural analgesia and reduce the number of epidural attempts, even by trainees [24]. It should be noted, however, that the depth predicted by ultrasonography can underestimate the true distance from the skin to the epidural or intrathecal space because of tissue compression by the probe for image optimization.

The accurate identification of the intervertebral level is also related to safety issues in neuraxial blockade. Ultrasonography more accurately determines the intervertebral level than palpation [17]. The level of the conus medullaris varies from T12 to L3 [25]. Although most studies on US-assisted neuraxial blockade evaluated safety outcomes as secondary outcome measures [26], preprocedural US imaging may help in avoiding conus medullaris injury, which can be caused by unintended dural puncture in the level above the L1-L2 interspace.

\section{UP-TO-DATE LITERATURE REVIEW}

The utility of US imaging in improving technical performance of neuraxial blockade has been evaluated in various patient populations. Table 1 shows the results of randomized controlled trials regarding the efficacy of US-guided neuraxial blockade compared with landmark-guided technique.

\section{Obstetric population}

Early studies on US-assisted neuraxial block were conducted in obstetric patients. In a series of randomized controlled trials from 2001 to 2002, Grau et al. [27,28] reported that preprocedural US imaging was associated with fewer needle passes and better analgesic efficacy in labor epidural analgesia. Additionally, for parturients with anticipated technical difficulty, including history of difficult epidural puncture, anatomical alteration of the lumbar spine, and body mass index $>33 \mathrm{~kg} / \mathrm{m}^{2}$, US assistance resulted in fewer needle passes, fewer puncture sites, lower pain score, and improved patient satisfaction [29].

However, more recent studies have shown inconsistent results. Nassar and Abdelazim [30] reported that US imaging increased the rate of successful procedure at the first attempt and reduced the number of needle passes compared to the palpation technique for combined epidural-spinal anesthesia (CSE). Perna et al. [31] also reported that US assistance enhanced technical performance of labor epidural analgesia, by providing anatomical information on the location of the intervertebral space, optimal needle insertion point, and tilting angle of the epidural needle. In contrast, other studies failed to highlight the benefits of preprocedural US imaging in obstetric patients [32-35]. Possible explanations for the conflicting results are 


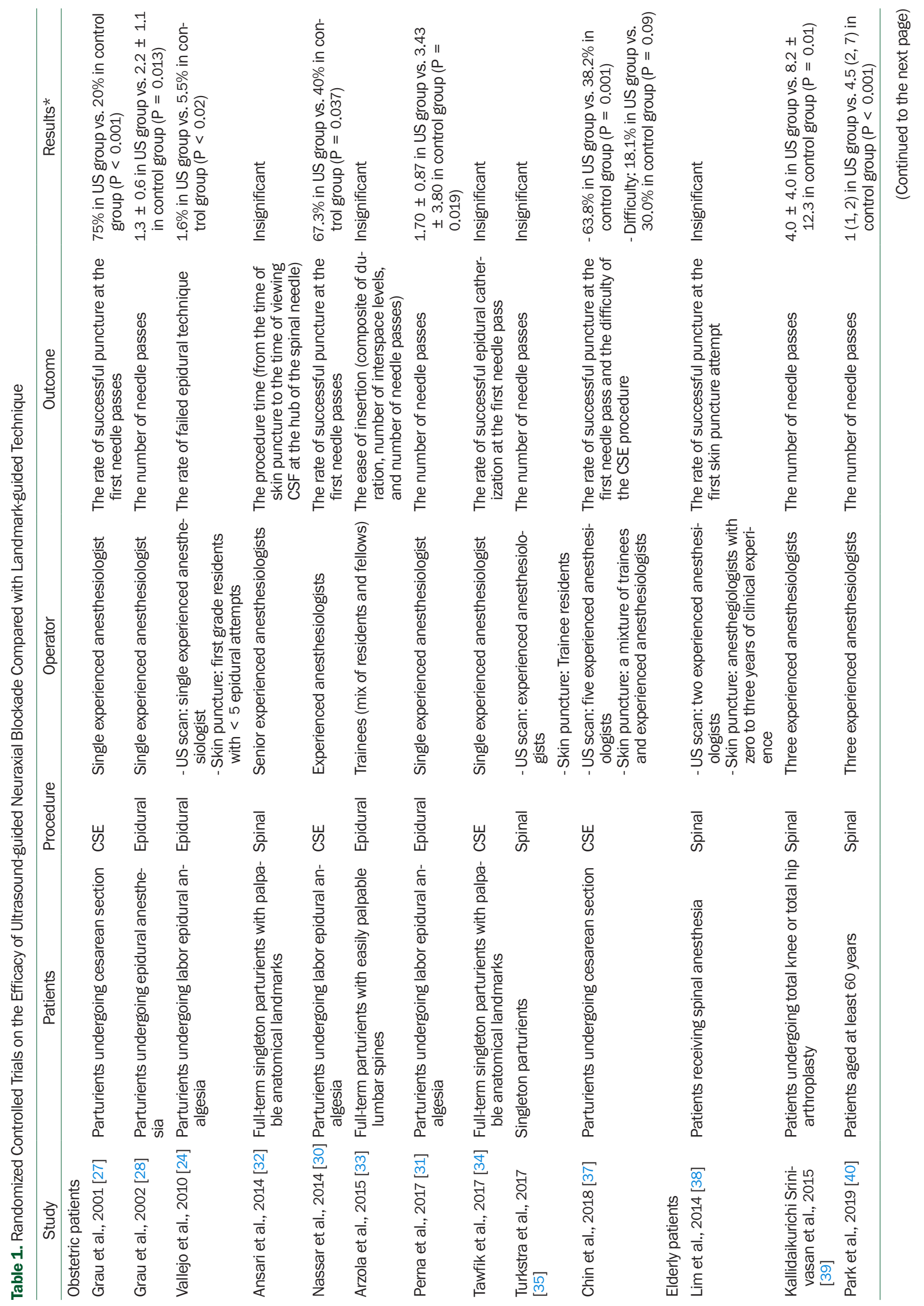




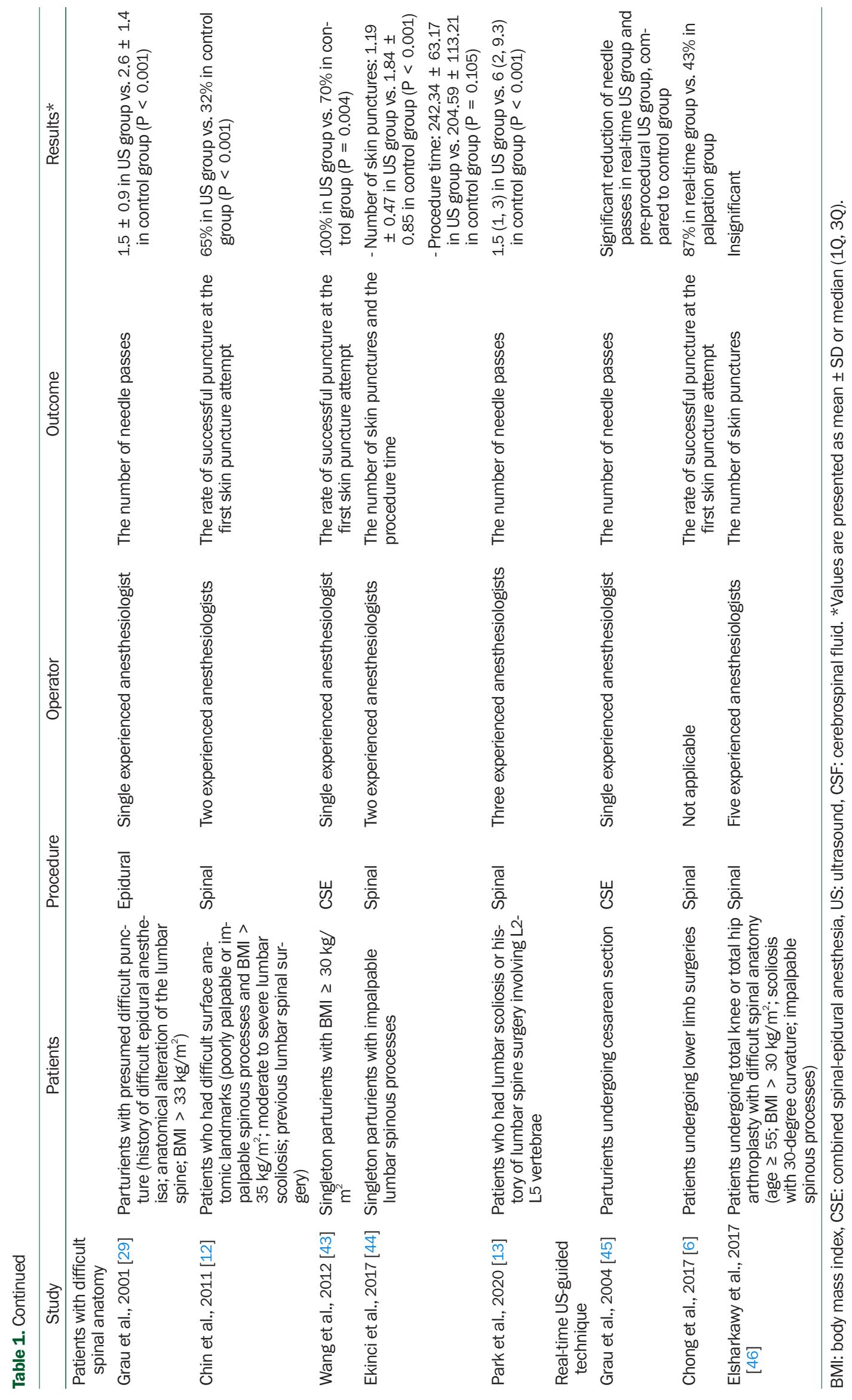


the characteristics of the study subjects and proceduralists. All these studies evaluated the utility of ultrasonography in parturients with palpable anatomical landmarks. In this population, the benefit of US imaging may be underestimated because neuraxial blockade is usually not complicated in lean patients or those who had normal vertebral anatomy. Regarding the proceduralists, experienced anesthesiologists performed the US scan and neuraxial blockade in two studies [32,34], while skin puncture was performed by trainees after ultrasonographic examination by experts in another study [35]. The guidance from a study investigator during skin puncture or suboptimal needle handling by the trainees may have led to the negative results [36]. However, in a recent large study conducted in women undergoing cesarean section with CSE, the authors found that US assistance improved technical performance in patients with easily palpable landmarks, but not in those with impalpable surface landmarks, and that the experience of proceduralists did not influence the first-pass success rate of CSE procedure [37]. Further studies are still needed to clarify which populations benefit the most through US assistance.

\section{Elderly patients}

The efficacy of US-assisted neuraxial blockade is more evident in elderly patients. In contrast to using the midline approach in obstetric patients, the paramedian approach was used in studies evaluating the utility of ultrasonography in the elderly. Lim et al. [38] compared the rate of successful dural puncture at the first attempt in patients receiving spinal anesthesia with or without preprocedural US imaging. Although the first-attempt success rate was not significantly different, shorter time was required to perform the procedure with US-assisted spinal anesthesia and patients were more satisfied compared to the manual palpation technique. Other studies showed consistent results that the number of needle passes and skin punctures were significantly decreased when using US-assisted spinal anesthesia, compared to the midline approach [39] or paramedian approach [40]. In general, neuraxial blockade is more difficult in an older population than in relatively younger obstetric patients, possibly due to degenerative changes of the lumbar spine, such as the calcified interspinous ligament and limited lumbar flexion [39]. These findings supported that preprocedural US imaging may be more beneficial in patients with difficult anatomy, as shown in a recent meta-analysis [41]. Scanning both sides and all spinal levels before selecting a puncture site for US-guided spinal anesthesia is recommended. The L5-S1 intervertebral level is a good option for neuraxial anesthesia in the elderly [42].

\section{Patients with difficult anatomy (obesity, scoliosis, or history of spine surgery)}

Several studies have evaluated whether US assistance improves technical performance of neuraxial blockade in patients with difficult anatomy, including moderate to severe obesity, lumbar scoliosis, ankylosing spondylitis, or history of lumbar spine surgery. Chin et al. [12] compared the first-attempt success rate of spinal anesthesia with or without US assistance in this population and found that preprocedural US imaging facilitates the performance of spinal anesthesia. Similar results were shown in obstetric patients with difficult anatomical landmarks. Wang et al. [43] reported that US scanning performed by single experienced anesthesiologist before neuraxial blockade significantly enhanced the first-attempt success rate. Another study published by Ekinci et al. [44] demonstrated that the number of skin punctures was significantly decreased when using preprocedural US imaging, but total procedure time was comparable with the conventional spinal anesthesia technique. Our recent study conducted in patients with documented lumbar scoliosis or those with history of previous spinal surgery also showed similar results that the number of needle passes and puncture attempts were significantly lower in the US group than in the control group, but total procedure time was not significantly different between the two groups [13]. Despite of US scanning time, difficulties in identifying the midline or intervertebral space in patients with abnormal vertebral anatomy would increase the procedural time in conventional palpation technique, resulting in no difference in the overall procedure time. Considering the reduced number of needle manipulations and better patient satisfaction, US neuraxial imaging should be accompanied in patients who are expected to have difficult neuraxial blockade.

\section{Real-time US-guided technique}

There are limited studies assessing the benefits of real-time US guidance technique. Grau et al. [45] compared real-time US-guided CSE procedure using the parasagittal 
oblique view with preprocedural US scanning and conventional landmark palpation technique and found that both US-guided techniques significantly reduced the number of needle passes. The advantage of real-time US guidance was also reported in a recent study by Chong et al. [6]. They found that first-attempt success rate was significantly higher when using real-time US-guided spinal anesthesia with the parasagittal oblique view, compared to the palpation-based paramedian approach [6]. However, another study on the efficacy of real-time US-guided spinal anesthesia in patients with difficult spinal anatomy showed no advantage of real-time technique over conventional landmark technique [46]. Various approaches, including transverse [8,47] and diagonal in-plane approaches [7], have been investigated for real-time US-guided neuraxial block. Despite some results showing the advantages of real-time US guidance, there are still technical challenges to be addressed, such as visualization of a small-gauge needle around the deep target structures.

\section{CONCLUSION}

For better clinical practice, it is recommended to apply US guidance for neuraxial blockade. US-guided neuraxial block can facilitate successful access to the intrathecal or epidural space in patients with difficult spinal anatomy, as well as in those with easily palpable anatomical landmarks. Anesthesiologists who routinely perform lumbar neuraxial block should be familiar with the sonoanatomy of the lumbar vertebrae and US-guided techniques to improve technical performance and safety.

\section{CONFLICTS OF INTEREST}

No potential conflict of interest relevant to this article was reported.

\section{AUTHOR CONTRIBUTIONS}

Conceptualization: Jin-Tae Kim. Data curation: Seokha Yoo, Youngwon Kim, Sun-Kyung Park, Sang-Hwan Ji. Methodology: Jin-Tae Kim. Project administration: Jin-Tae Kim. Writing-original draft: Seokha Yoo, Jin-Tae Kim. Writing-review \& editing: Seokha Yoo, Youngwon Kim, Sun-Kyung Park, Sang-Hwan Ji, Jin-Tae Kim. Investigation: Seokha Yoo, Youngwon Kim, Sun-Kyung Park, Sang-Hwan Ji.

\section{ORCID}

Seokha Yoo, https://orcid.org/0000-0003-4679-6027

Youngwon Kim, https://orcid.org/0000-0002-1071-5494

Sun-Kyung Park, https://orcid.org/0000-0002-4670-253X

Sang-Hwan Ji, https://orcid.org/0000-0001-6736-4464

Jin-Tae Kim, https://orcid.org/0000-0002-3738-0081

\section{REFERENCES}

1. Chin KJ, Karmakar MK, Peng P. Ultrasonography of the adult thoracic and lumbar spine for central neuraxial blockade. Anesthesiology 2011; 114: 1459-85.

2. Pintaric TS, Hadzic A, Strbenc M, Podpecan O, Podbregar M, Cvetko E. Inflammatory response after injection of aqueous gel into subarachnoid space in piglets. Reg Anesth Pain Med 2013; 38: $100-5$.

3. Killeen T, Kamat A, Walsh D, Parker A, Aliashkevich A. Severe adhesive arachnoiditis resulting in progressive paraplegia following obstetric spinal anaesthesia: a case report and review. Anaesthesia 2012; 67: 1386-94.

4. Tran D, Kamani AA, Al-Attas E, Lessoway VA, Massey S, Rohling RN. Single-operator real-time ultrasound-guidance to aim and insert a lumbar epidural needle. Can J Anaesth 2010; 57: 313-21.

5. Chin KJ, Chan VW, Ramlogan R, Perlas A. Real-time ultrasound-guided spinal anesthesia in patients with a challenging spinal anatomy: two case reports. Acta Anaesthesiol Scand 2010; 54: 252-5.

6. Chong SE, Mohd Nikman A, Saedah A, Wan Mohd Nazaruddin WH, Kueh YC, Lim JA, et al. Real-time ultrasound-guided paramedian spinal anaesthesia: evaluation of the efficacy and the success rate of single needle pass. Br J Anaesth 2017; 118: 799801.

7. Conroy PH, Luyet C, McCartney CJ, McHardy PG. Real-time ultrasound-guided spinal anaesthesia: a prospective observational study of a new approach. Anesthesiol Res Pract 2013; 2013: 525818 .

8. Liu Y, Qian W, Ke XJ, Mei W. Real-time ultrasound-guided spinal anesthesia using a new paramedian transverse approach. Curr Med Sci 2018; 38: 910-3.

9. Niazi AU, Chin KJ, Jin R, Chan VW. Real-time ultrasound-guided spinal anesthesia using the SonixGPS ultrasound guidance system: a feasibility study. Acta Anaesthesiol Scand 2014; 58: 875-81.

10. Bowens C, Dobie KH, Devin CJ, Corey JM. An approach to neuraxial anaesthesia for the severely scoliotic spine. Br J An- 
aesth 2013; 111: 807-11.

11. Ko JY, Leffert LR. Clinical implications of neuraxial anesthesia in the parturient with scoliosis. Anesth Analg 2009; 109: 19304 .

12. Chin KJ, Perlas A, Chan V, Brown-Shreves D, Koshkin A, Vaishnav $V$. Ultrasound imaging facilitates spinal anesthesia in adults with difficult surface anatomic landmarks. Anesthesiology 2011; 115: 94-101.

13. Park SK, Bae J, Yoo S, Kim WH, Lim YJ, Bahk JH, et al. Ultrasound-assisted versus landmark-guided spinal anesthesia in patients with abnormal spinal anatomy: a randomized controlled trial. Anesth Analg 2020; 130: 787-95.

14. Whitty R, Moore M, Macarthur A. Identification of the lumbar interspinous spaces: palpation versus ultrasound. Anesth Analg 2008; 106: 538-40.

15. Chin KJ, Perlas A, Singh M, Arzola C, Prasad A, Chan V, et al. An ultrasound-assisted approach facilitates spinal anesthesia for total joint arthroplasty. Can J Anaesth 2009; 56: 643-50.

16. Watson MJ, Evans S, Thorp JM. Could ultrasonography be used by an anaesthetist to identify a specified lumbar interspace before spinal anaesthesia? Br J Anaesth 2003; 90: 509-11.

17. Furness G, Reilly MP, Kuchi S. An evaluation of ultrasound imaging for identification of lumbar intervertebral level. Anaesthesia 2002; 57: 277-80.

18. Balki M. Locating the epidural space in obstetric patients-ultrasound a useful tool: continuing professional development. Can J Anaesth 2010; 57: 1111-26.

19. Chin KJ, Ramlogan R, Arzola C, Singh M, Chan V. The utility of ultrasound imaging in predicting ease of performance of spinal anesthesia in an orthopedic patient population. Reg Anesth Pain Med 2013; 38: 34-8.

20. Gnaho A, Nguyen V, Villevielle T, Frota M, Marret E, Gentili ME. Assessing the depth of the subarachnoid space by ultrasound. Rev Bras Anestesiol 2012; 62: 520-30.

21. Helayel PE, da Conceição DB, Meurer G, Swarovsky C, de Oliveira Filho GR. Evaluating the depth of the epidural space with the use of ultrasound. Rev Bras Anestesiol 2010; 60: 376-82.

22. Balki M, Lee Y, Halpern S, Carvalho JC. Ultrasound imaging of the lumbar spine in the transverse plane: the correlation between estimated and actual depth to the epidural space in obese parturients. Anesth Analg 2009; 108: 1876-81.

23. Arzola C, Davies S, Rofaeel A, Carvalho JC. Ultrasound using the transverse approach to the lumbar spine provides reliable landmarks for labor epidurals. Anesth Analg 2007; 104: 118892.

24. Vallejo MC, Phelps AL, Singh S, Orebaugh SL, Sah N. Ultrasound decreases the failed labor epidural rate in resident train- ees. Int J Obstet Anesth 2010; 19: 373-8.

25. Kim JT, Bahk JH, Sung J. Influence of age and sex on the position of the conus medullaris and Tuffier's line in adults. Anesthesiology 2003; 99: 1359-63.

26. Neal JM, Brull R, Horn JL, Liu SS, McCartney CJ, Perlas A, et al. The second American Society of Regional Anesthesia and Pain Medicine evidence-based medicine assessment of ultrasound-guided regional anesthesia: executive summary. Reg Anesth Pain Med 2016; 41: 181-94.

27. Grau T, Leipold RW, Conradi R, Martin E, Motsch J. Ultrasound imaging facilitates localization of the epidural space during combined spinal and epidural anesthesia. Reg Anesth Pain Med 2001; 26: 64-7.

28. Grau T, Leipold RW, Conradi R, Martin E, Motsch J. Efficacy of ultrasound imaging in obstetric epidural anesthesia. J Clin Anesth 2002; 14: 169-75.

29. Grau T, Leipold RW, Conradi R, Martin E. Ultrasound control for presumed difficult epidural puncture. Acta Anaesthesiol Scand 2001; 45: 766-71.

30. Nassar M, Abdelazim IA. Pre-puncture ultrasound guided epidural insertion before vaginal delivery. J Clin Monit Comput 2015; 29: 573-7.

31. Perna P, Gioia A, Ragazzi R, Volta CA, Innamorato M. Can pre-procedure neuroaxial ultrasound improve the identification of the potential epidural space when compared with anatomical landmarks? A prospective randomized study. Minerva Anestesiol 2017; 83: 41-9.

32. Ansari T, Yousef A, El Gamassy A, Fayez M. Ultrasound-guided spinal anaesthesia in obstetrics: is there an advantage over the landmark technique in patients with easily palpable spines? Int J Obstet Anesth 2014; 23: 213-6.

33. Arzola C, Mikhael R, Margarido C, Carvalho JC. Spinal ultrasound versus palpation for epidural catheter insertion in labour: a randomised controlled trial. Eur J Anaesthesiol 2015; 32: 499-505.

34. Tawfik MM, Atallah MM, Elkharboutly WS, Allakkany NS, Abdelkhalek M. Does preprocedural ultrasound increase the firstpass success rate of epidural catheterization before cesarean delivery? A randomized controlled trial. Anesth Analg 2017; 124: 851-6.

35. Turkstra TP, Marmai KL, Armstrong KP, Kumar K, Singh SI. Preprocedural ultrasound assessment does not improve trainee performance of spinal anesthesia for obstetrical patients: a randomized controlled trial. J Clin Anesth 2017; 37: 21-4.

36. Chin KJ. Recent developments in ultrasound imaging for neuraxial blockade. Curr Opin Anaesthesiol 2018; 31: 608-13.

37. Chin A, Crooke B, Heywood L, Brijball R, Pelecanos AM, Abey- 
pala W. A randomised controlled trial comparing needle movements during combined spinal-epidural anaesthesia with and without ultrasound assistance. Anaesthesia 2018; 73: 466-73.

38. Lim YC, Choo CY, Tan KT. A randomised controlled trial of ultrasound-assisted spinal anaesthesia. Anaesth Intensive Care 2014; 42: 191-8.

39. Kallidaikurichi Srinivasan K, Iohom G, Loughnane F, Lee PJ. Conventional landmark-guided midline versus preprocedure ultrasound-guided paramedian techniques in spinal anesthesia. Anesth Analg 2015; 121: 1089-96.

40. Park SK, Yoo S, Kim WH, Lim YJ, Bahk JH, Kim JT. Ultrasound-assisted vs. landmark-guided paramedian spinal anaesthesia in the elderly: a randomised controlled trial. Eur J Anaesthesiol 2019; 36: 763-71.

41. Jiang L, Zhang F, Wei N, Lv J, Chen W, Dai Z. Could preprocedural ultrasound increase the first-pass success rate of neuraxial anesthesia in obstetrics? A systematic review and meta-analysis of randomized controlled trials. J Anesth 2020; 34: 434-44.

42. Bae J, Park SK, Yoo S, Lim YJ, Kim JT. Influence of age, laterality, patient position, and spinal level on the interlamina space for spinal puncture. Reg Anesth Pain Med 2020; 45: 27-31.

43. Wang Q, Yin C, Wang TL. Ultrasound facilitates identification of combined spinal-epidural puncture in obese parturients. Chin Med J (Engl) 2012; 125: 3840-3.

44. Ekinci M, Alici HA, Ahiskalioglu A, Ince I, Aksoy M, Celik EC, et al. The use of ultrasound in planned cesarean delivery under spinal anesthesia for patients having nonprominent anatomic landmarks. J Clin Anesth 2017; 37: 82-5.

45. Grau T, Leipold RW, Fatehi S, Martin E, Motsch J. Real-time ultrasonic observation of combined spinal-epidural anaesthesia. Eur J Anaesthesiol 2004; 21: 25-31.

46. Elsharkawy H, Maheshwari A, Babazade R, Perlas A, Zaky S, Mounir-Soliman L. Real-time ultrasound-guided spinal anesthesia in patients with predicted difficult anatomy. Minerva Anestesiol 2017; 83: 465-73.

47. Elsharkawy H, Saasouh W, Babazade R, Soliman LM, Horn JL, Zaky S. Real-time ultrasound-guided lumbar epidural with transverse interlaminar view: evaluation of an in-plane technique. Pain Med 2019; 20: 1750-5. 